\title{
Idéaux fermés de certaines algèbres de Beurling et application aux opérateurs à spectre dénombrable \\ par
}

\author{
Cyril Agrafeuil (Bordeaux)
}

\begin{abstract}
We denote by $\mathbb{T}$ the unit circle and by $\mathbb{D}$ the unit disc of $\mathbb{C}$. Let $s$ be a non-negative real and $\omega$ a weight such that $\omega(n)=(1+n)^{s}(n \geq 0)$ and the sequence $\left(\omega(-n) /(1+n)^{s}\right)_{n \geq 0}$ is non-decreasing. We define the Banach algebra

$$
A_{\omega}(\mathbb{T})=\left\{f \in \mathcal{C}(\mathbb{T}):\|f\|_{\omega}=\sum_{n=-\infty}^{+\infty}|\widehat{f}(n)| \omega(n)<+\infty\right\} .
$$

If $I$ is a closed ideal of $A_{\omega}(\mathbb{T})$, we set $h^{0}(I)=\{z \in \mathbb{T}: f(z)=0(f \in I)\}$. We describe all closed ideals $I$ of $A_{\omega}(\mathbb{T})$ such that $h^{0}(I)$ is at most countable. A similar result is obtained for closed ideals of the algebra $A_{s}^{+}(\mathbb{T})=\left\{f \in A_{\omega}(\mathbb{T}): \widehat{f}(n)=0(n<0)\right\}$ without inner factor. Then we use this description to establish a link between operators with countable spectrum and interpolating sets for $a^{\infty}$, the space of infinitely differentiable functions in the closed unit disc $\overline{\mathbb{D}}$ and holomorphic in $\mathbb{D}$.
\end{abstract}

1. Introduction. On note $\mathbb{T}$ le cercle unité et $\mathbb{D}$ le disque unité. Pour un entier $p \geq 0$, on note $\mathcal{C}^{p}(\mathbb{T})$ l'algèbre des fonctions $p$ fois continûment dérivables sur $\mathbb{T}$. Si $\omega=(\omega(n))_{n \in \mathbb{Z}}$ est une suite de réels strictement positifs, on définit

$$
A_{\omega}(\mathbb{T})=\left\{f \in \mathcal{C}^{0}(\mathbb{T}):\|f\|_{\omega}=\sum_{n=-\infty}^{+\infty}|\widehat{f}(n)| \omega(n)<+\infty\right\},
$$

où $\widehat{f}(n)$ désigne le $n^{\text {ième }}$ coefficient de Fourier de $f$. On dit qu'une suite $\omega=(\omega(n))_{n \in \mathbb{Z}}$ est un poids si $\omega(n) \geq 1$ et $\omega(m+n) \leq \omega(m) \omega(n)$ pour tout $m, n$ dans $\mathbb{Z}$. Si $\omega$ est un poids, $A_{\omega}(\mathbb{T})$ muni de la norme \|\|$_{\omega}$ est une algèbre de Banach. Elle est régulière si et seulement si

$$
\sum_{n=-\infty}^{+\infty} \frac{\log \omega(n)}{1+n^{2}}<+\infty
$$

(voir $\left[12\right.$, ex. 7 , p. 118]). Soit $p$ un entier positif tel que $A_{\omega}(\mathbb{T}) \subset \mathcal{C}^{p}(\mathbb{T})$. Si $I$ est un idéal fermé de $A_{\omega}(\mathbb{T})$, on pose

2000 Mathematics Subject Classification: 46J20, 47A30, 30H05. 


$$
h^{k}(I)=\left\{z \in \mathbb{T}: f(z)=\cdots=f^{(k)}(z)=0(f \in I)\right\} \quad(k \in\{0, \ldots, p\}) .
$$

Soit $s$ un réel positif; on note $[s]$ sa partie entière. On dira qu'une suite de réels strictement positifs $\omega=(\omega(n))_{n \in \mathbb{Z}}$ vérifie la condition $\left(\mathrm{W}_{s}\right)$ si

$$
\left\{\begin{array}{l}
\omega(n)=(1+n)^{s} \quad(n \geq 0), \\
\text { la suite }\left(\frac{\omega(-n)}{(1+n)^{s}}\right)_{n \geq 0} \text { est croissante }
\end{array}\right.
$$

et qu'elle vérifie la condition $(\mathrm{A})$ si

$$
\omega(-n)=O\left(e^{\varepsilon \sqrt{n}}\right) \quad(n \rightarrow+\infty) \text { pour tout } \varepsilon>0 .
$$

Soit $\omega=(\omega(n))_{n \in \mathbb{Z}}$ un poids vérifiant les conditions $\left(\mathrm{W}_{s}\right)$ et $(\mathrm{A})$. On a alors l'inclusion $A_{\omega}(\mathbb{T}) \subset \mathcal{C}^{[s]}(\mathbb{T})$, et $A_{\omega}(\mathbb{T})$ est une algèbre de Banach régulière. Dans le cas particulier où le poids $\omega$ est défini par $\omega(n)=(1+|n|)^{s}$ pour tout $n \in \mathbb{Z}$, avec $s$ un réel positif, on notera $\left(A_{s}(\mathbb{T}),\|\|_{s}\right)$ l'algèbre $\left(A_{\omega}(\mathbb{T}),\|\|_{\omega}\right)$. On remarque que $A_{0}(\mathbb{T})=A(\mathbb{T})$ n'est rien d'autre que l'algèbre de Wiener. On posera également

$$
A_{s}^{+}(\mathbb{T})=\left\{f \in A_{s}(\mathbb{T}): \widehat{f}(n)=0(n<0)\right\} .
$$

Pour $f \in A_{s}^{+}(\mathbb{T})$, on note $S(f)$ son facteur intérieur et on pose

$$
Z_{+}^{k}(f)=\left\{z \in \overline{\mathbb{D}}: f(z)=\cdots=f^{(k)}(z)=0\right\} \quad(k \in\{0, \ldots,[s]\}) .
$$

Si $I$ est un idéal fermé de $A_{s}^{+}(\mathbb{T})$, on note $S_{I}$ son facteur intérieur, c'est-à-dire le plus grand diviseur intérieur commun à tous les éléments de $I$ non nuls (voir [10, p. 85]), et on pose

$$
h_{+}^{k}(I)=\bigcap_{f \in I} Z_{+}^{k}(f) .
$$

Nous décrivons dans un premier temps tous les idéaux fermés $I$ de $A_{\omega}(\mathbb{T})$ lorsque $h^{0}(I)$ est au plus dénombrable et lorsque le poids $\omega$ vérifie les conditions $\left(\mathrm{W}_{s}\right)$ et $(\mathrm{A})$. Plus précisément, nous montrons (théorème 3.2) que, sous ces conditions, on a

$$
I=\left\{f \in A_{\omega}(\mathbb{T}): f^{(j)}(z)=0 \operatorname{sur} h^{j}(I)(0 \leq j \leq[s])\right\} .
$$

Dans le cas $s=0$, nous retrouvons ainsi un résultat connu (voir [19]). Nous déduisons alors de ce résultat une caractérisation des idéaux fermés $I$ de $A_{s}^{+}(\mathbb{T})$ sans facteur intérieur (c'est-à-dire tels que $S_{I}=1$ ) tels que $h_{+}^{0}(I)$ est au plus dénombrable. On note $\mathcal{H}^{\infty}(\mathbb{D})$ l'algèbre des fonctions holomorphes et bornées dans $\mathbb{D}$. Si $E_{[s]} \subset \cdots \subset E_{0}$ sont des fermés de $\mathbb{T}$ et $S$ une fonction intérieure, on définit

$$
\begin{aligned}
& I\left(S ; E_{0}, \ldots, E_{[s]}\right) \\
& \quad=\left\{f \in A_{s}^{+}(\mathbb{T}): S \mid S(f), E_{0} \subset Z_{+}^{0}(f) \cap \mathbb{T}, \ldots, E_{[s]} \subset Z_{+}^{[s]}(f) \cap \mathbb{T}\right\},
\end{aligned}
$$


où $S \mid S(f)$ signifie que $S$ divise $S(f)$, c'est-à-dire que $S(f) / S$ est dans $\mathcal{H}^{\infty}(\mathbb{D})$. Nous montrons que si $I$ est un idéal fermé de $A_{s}^{+}(\mathbb{T})$ sans facteur intérieur tel que $h_{+}^{0}(I)$ est au plus dénombrable, alors

$$
I=I\left(1 ; h_{+}^{0}(I) \cap \mathbb{T}, \ldots, h_{+}^{[s]}(I) \cap \mathbb{T}\right) .
$$

Notons que dans le cas $s=0$, les idéaux fermés $I$ de $A^{+}(\mathbb{T})=A_{0}^{+}(\mathbb{T})$ ont été caractérisés par J.-P. Kahane dans [11] lorsque $h_{+}^{0}(I)$ est fini, par C. Bennett et J. E. Gilbert dans [3] lorsque $h_{+}^{0}(I)$ est dénombrable, et enfin par J. Esterle, E. Strouse et F. Zouakia dans [8] lorsque $h_{+}^{0}(I)$ est le Cantor triadique. D'autre part, J. Esterle a construit un idéal fermé $I$ de $A^{+}(\mathbb{T})$ tel que $I \neq I\left(S_{I} ; h_{+}^{0}(I)\right)$, démontrant ainsi que la conjecture de C. Bennett et J. E. Gilbert dans [3] est fausse.

Dans un deuxième temps, nous allons utiliser ce résultat pour étudier le comportement de certains opérateurs à spectre dénombrable et inclus dans $\mathbb{T}$. Soit $E$ un fermé de $\mathbb{T}$ et $s, t$ deux réels positifs ou nuls. On désignera par $P(s, t, E)$ la propriété suivante : tout opérateur $T$ inversible sur un espace de Banach tel que $\operatorname{Sp} T \subset E$ et qui vérifie les conditions

$$
\begin{aligned}
\left\|T^{n}\right\| & =O\left(n^{s}\right) & & (n \rightarrow+\infty), \\
\left\|T^{-n}\right\| & =O\left(e^{\varepsilon \sqrt{n}}\right) & & (n \rightarrow+\infty) \text { pour tout } \varepsilon>0,
\end{aligned}
$$

vérifie également la propriété plus forte

$$
\left\|T^{-n}\right\|=O\left(n^{t}\right) \quad(n \rightarrow+\infty) .
$$

M. Zarrabi a montré dans [19, théorème 3.1 et remarque 2.a] qu'un fermé $E$ de $\mathbb{T}$ vérifie la propriété $P(0,0, E)$ si et seulement si $E$ est dénombrable.

Nous nous proposons d'étudier la propriété $P(s, t, E)$ pour n'importe quel réel $s \geq 0$. On dira qu'un fermé $E$ de $\mathbb{T}$ vérifie la condition de Carleson si

$$
\int_{0}^{2 \pi} \log ^{+} \frac{1}{d\left(e^{i t}, E\right)} d t<+\infty
$$

et qu'il vérifie la condition (ATW) s'il existe des constantes $C_{1}, C_{2}>0$ telles que

$$
\frac{1}{|L|} \int_{L} \log ^{+} \frac{1}{d\left(e^{i t}, E\right)} d t \leq C_{1} \log \frac{1}{|L|}+C_{2} \quad \text { pour tout } \operatorname{arc} L \text { de } \mathbb{T},
$$

où $|L|$ désigne la longueur de l'arc $L$, et $d\left(e^{i t}, E\right)$ la distance de $e^{i t}$ à $E$.

La condition (ATW) vient de [1], où les auteurs montrent que les ensembles vérifiant (ATW) sont les ensembles d'interpolation pour $a^{\infty}$, l'espace des fonctions holomorphes dans le disque unité et de classe $\mathcal{C}^{\infty}$ dans $\overline{\mathbb{D}}$.

Nous montrons (théorème 5.2) que si $E$ est un fermé dénombrable de $\mathbb{T}$, alors les deux assertions suivantes sont équivalentes : 
(i) $E$ vérifie la condition (ATW).

(ii) $E$ vérifie la condition (C) et pour tout réel $s \geq 0$, il existe un réel $t$ tel que la propriété $P(s, t, E)$ soit vérifiée.

Nous montrons ensuite que si $E$ n'est pas dénombrable, alors la propriété $P(0, t, E)$ n'est vérifiée pour aucun réel $t \geq 0$.

Je voudrais remercier mon directeur de thèse, Mohamed Zarrabi, pour les nombreuses discussions que j'ai eues avec lui, et qui sont à l'origine de cet article.

2. Unité approchée pour certains idéaux de $A_{\omega}(\mathbb{T})$. Nous allons établir ici que lorsque le poids $\omega$ satisfait la condition $\left(W_{s}\right)$, alors l'algèbre $A_{\omega}(\mathbb{T})$ vérifie la condition de Ditkin analytique forte (au sens de $[3$, p. 4]). Pour cela, nous avons besoin des trois lemmes élémentaires suivants :

Lemme 2.1. Soient $\beta$ un réel tel que $0 \leq \beta<1$ et $j$ un entier positif. Alors pour tout réel $x$ tel que $0 \leq x<1$, nous avons l'inégalité suivante:

$$
\sum_{k=j}^{+\infty}(1+k)^{\beta} x^{k} \leq \frac{(j+1)^{\beta} x^{j}}{1-x}+\frac{x^{j+1}}{(1-x)^{\beta+1}} .
$$

Démonstration. Soit $x$ un réel tel que $0 \leq x<1$; posons

$$
f(x)=\sum_{k=j}^{+\infty}(1+k)^{\beta} x^{k}-\frac{(j+1)^{\beta} x^{j}}{1-x}-\frac{x^{j+1}}{(1-x)^{\beta+1}} .
$$

En développant en série les fonctions $x \mapsto 1 /(1-x)$ et $x \mapsto 1 /(1-x)^{\beta+1}$, on montre que

$$
f(x)=\sum_{k=1}^{+\infty} a_{k} x^{k+j},
$$

avec $a_{1}=(j+2)^{\beta}-(j+1)^{\beta}-1$ et pour tout $k \geq 2$,

$$
a_{k}=(k+j+1)^{\beta}-(j+1)^{\beta}-\frac{(\beta+1) \cdots(\beta+k-1)}{(k-1) !} .
$$

Il est facile de voir que $a_{1} \leq 0$. Pour démontrer ce lemme, il suffit donc de montrer que pour tout $k \geq 2, a_{k} \leq 0$. On commence par remarquer que, pour tout $k \geq 2$, la fonction

$$
t \mapsto(k+t+1)^{\beta}-(t+1)^{\beta}-\frac{(\beta+1) \cdots(\beta+k-1)}{(k-1) !}
$$

est décroissante sur $[-1,+\infty)$. Par conséquent, pour tout $k \geq 2$, on a

$$
a_{k} \leq k^{\beta}-\frac{(\beta+1) \cdots(\beta+k-1)}{(k-1) !} .
$$


Puisque $\beta<1$, on a $(1+t)^{\beta} \leq 1+\beta t$ pour tout réel $t$ positif. En utilisant cette inégalité, on obtient

$$
\begin{aligned}
\frac{(\beta+1) \cdots(\beta+k-1)}{(k-1) !} & =(1+\beta)\left(1+\frac{\beta}{2}\right) \cdots\left(1+\frac{\beta}{k-1}\right) \\
& \geq(1+1)^{\beta}\left(1+\frac{1}{2}\right)^{\beta} \cdots\left(1+\frac{1}{k-1}\right)^{\beta}=k^{\beta} .
\end{aligned}
$$

On déduit alors de cette inégalité et de (4) que $a_{k} \leq 0$ pour tout $k \geq 2$, ce qu'il s'agissait de démontrer.

Lemme 2.2. Soit $\omega=(\omega(n))_{n \in \mathbb{Z}}$ une suite de réels strictement positifs telle que:

(i) les suites $(\omega(-n) /(1+n))_{n \geq 0}$ et $(\omega(n) /(1+n))_{n \geq 0}$ soient croissantes.

(ii) $0<\inf _{n \in \mathbb{Z}} \frac{\omega(n+1)}{\omega(n)}<\sup _{n \in \mathbb{Z}} \frac{\omega(n+1)}{\omega(n)}<+\infty$.

On note $A=\inf _{n \in \mathbb{Z}} \omega(n+1) / \omega(n)$ et $B=\sup _{n \in \mathbb{Z}} \omega(n+1) / \omega(n)$, et on définit la suite $\omega_{1}=\left(\omega_{1}(n)\right)_{n \in \mathbb{Z}}$ par

$$
\omega_{1}(n)=\frac{\omega(n)}{1+|n|} \quad(n \in \mathbb{Z}) .
$$

Alors on a les deux propriétés suivantes:

(1) On a la double inégalité $A\left\|f^{\prime}\right\|_{\omega_{1}} \leq\|f\|_{\omega} \leq|\widehat{f}(0)| \omega(0)+3 B\left\|f^{\prime}\right\|_{\omega_{1}}$.

(2) Soit $f$ dans $A_{\omega}(\mathbb{T})$ qui s'annule au point 1 ; alors $f /(\alpha-1)$ est dans $A_{\omega_{1}}(\mathbb{T})$, où $\alpha: z \mapsto z$.

Démonstration. (1) Cela découle immédiatement de la relation $\widehat{f}^{\prime}(n)=$ $(n+1) \widehat{f}(n+1)(n \in \mathbb{Z})$.

(2) On écrit $f=\sum_{n=-\infty}^{+\infty} \widehat{f}(n)\left(\alpha^{n}-1\right)$, d'où

$$
\frac{f}{\alpha-1}=-\sum_{n=-\infty}^{-1} \widehat{f}(n)\left(\alpha^{n}+\cdots+\alpha^{-1}\right)+\sum_{n=1}^{+\infty} \widehat{f}(n)\left(1+\cdots+\alpha^{n-1}\right) .
$$

Ainsi

$$
\begin{aligned}
\left\|\frac{f}{\alpha-1}\right\|_{\omega_{1}}= & \sum_{n=-\infty}^{-1}|\widehat{f}(n)|\left(\omega_{1}(n)+\cdots+\omega_{1}(-1)\right) \\
& +\sum_{n=1}^{+\infty}|\widehat{f}(n)|\left(\omega_{1}(0)+\cdots+\omega_{1}(n-1)\right) .
\end{aligned}
$$


Comme les suites $\left(\omega_{1}(-n)\right)_{n \geq 0}$ et $\left(\omega_{1}(n)\right)_{n \geq 0}$ sont croissantes, on déduit de (5) que

$$
\begin{aligned}
\left\|\frac{f}{\alpha-1}\right\|_{\omega_{1}} & \leq \sum_{n=-\infty}^{-1}|\widehat{f}(n)||n| \omega_{1}(n)+\sum_{n=1}^{+\infty}|\widehat{f}(n)| n \omega_{1}(n) \\
& \leq \sum_{n=-\infty}^{+\infty}|\widehat{f}(n)| \omega(n)<+\infty .
\end{aligned}
$$

Lemme 2.3. Soient $0 \leq \beta<1$ un réel et $\omega=(\omega(n))_{n \in \mathbb{Z}}$ une suite vérifiant la condition $\left(\mathrm{W}_{\beta}\right)$. On définit une suite de fonctions de $A_{\omega}(\mathbb{T})$ par

$$
e_{n}=\frac{\alpha-1}{\alpha-1-1 / n} \quad(n \geq 1),
$$

où $\alpha$ est la fonction $z \mapsto z$. Alors pour toute fonction $f$ dans $A_{\omega}(\mathbb{T})$ telle que $f(1)=0$, on a

$$
\begin{gathered}
\left\|\left(e_{n}-1\right) f\right\|_{\omega} \leq 3\|f\|_{\omega}, \\
\lim _{n \rightarrow+\infty}\left\|\left(e_{n}-1\right) f\right\|_{\omega}=0 .
\end{gathered}
$$

Démonstration. Soit $f$ dans $A_{\omega}(\mathbb{T})$ telle que $f(1)=0$. En écrivant $f=$ $f-f(1)=\sum_{j=-\infty}^{+\infty} \widehat{f}(j)\left(\alpha^{j}-1\right)$, on voit que (7) sera démontré si on vérifie que

$$
\left\|\left(e_{n}-1\right)\left(\alpha^{j}-1\right)\right\|_{\omega} \leq 3 \omega(j) \quad(j \in \mathbb{Z}) .
$$

Soit $n \geq 1$. Un simple calcul montre que

$$
e_{n}=1-\frac{1}{n+1} \sum_{k=0}^{+\infty}\left(\frac{n}{n+1}\right)^{k} \alpha^{k}
$$

et

$$
\left(e_{n}-1\right)\left(\alpha^{j}-1\right)=-\frac{1}{n+1} \sum_{k=0}^{+\infty}\left(\frac{n}{n+1}\right)^{k} \alpha^{j+k}+\frac{1}{n+1} \sum_{k=0}^{+\infty}\left(\frac{n}{n+1}\right)^{k} \alpha^{k} .
$$

Supposons que $j \geq 0$. On a

$$
\begin{aligned}
\left\|\left(e_{n}-1\right)\left(\alpha^{j}-1\right)\right\|_{\omega}= & \frac{1}{n+1} \sum_{k=0}^{j-1}\left(\frac{n}{n+1}\right)^{k}(1+k)^{\beta} \\
& +\frac{((n+1) / n)^{j}-1}{n+1} \sum_{k=j}^{+\infty}\left(\frac{n}{n+1}\right)^{k}(1+k)^{\beta} .
\end{aligned}
$$

On a alors l'inégalité suivante :

$$
\left(\frac{n+1}{n}\right)^{j}-1 \leq \min \left(1, \frac{j}{n}\right)\left(\frac{n+1}{n}\right)^{j} .
$$


En effet, $((n+1) / n)^{j}-1 \leq((n+1) / n)^{j}$, ce qui prouve l'inégalité ci-dessus dans le cas où $j \geq n$. Dans le cas où $j \leq n$ et $j \neq 0$, on déduit de l'inégalité des accroissements finis que

$$
\left(\frac{n+1}{n}\right)^{j}-1 \leq \frac{j}{n}\left(\frac{n+1}{n}\right)^{j-1}=\frac{j}{n+1}\left(\frac{n+1}{n}\right)^{j}
$$

Par conséquent, dans le cas $j \leq n$, on a

$$
\left(\frac{n+1}{n}\right)^{j}-1 \leq \frac{j}{n}\left(\frac{n+1}{n}\right)^{j}
$$

(le cas $j=0$ étant évident). On déduit alors de l'inégalité (9) et du lemme 2.1 appliqué pour $x=n /(n+1)$ la majoration suivante :

$$
\begin{aligned}
\left\|\left(e_{n}-1\right)\left(\alpha^{j}-1\right)\right\|_{\omega} \leq & \frac{1}{n+1} \sum_{k=0}^{j-1}\left(\frac{n}{n+1}\right)^{k}(1+k)^{\beta} \\
& +\min \left(1, \frac{j}{n}\right)\left((j+1)^{\beta}+(n+1)^{\beta}\right) .
\end{aligned}
$$

En observant maintenant que

$$
\frac{1}{n+1} \sum_{k=0}^{j-1}\left(\frac{n}{n+1}\right)^{k}(1+k)^{\beta} \leq(1+j)^{\beta},
$$

et en distinguant les cas $j \leq n$ et $j \geq n+1$, on obtient

$$
\left\|\left(e_{n}-1\right)\left(\alpha^{j}-1\right)\right\|_{\omega} \leq 3(1+j)^{\beta}=3 \omega(j) .
$$

Supposons maintenant que $j \leq-1$. On a

$$
\begin{aligned}
\left\|\left(e_{n}-1\right)\left(\alpha^{j}-1\right)\right\|_{\omega}= & \frac{1}{n+1} \sum_{k=j}^{-1}\left(\frac{n}{n+1}\right)^{k-j} \omega(k) \\
& +\frac{1-(n /(n+1))^{-j}}{n+1} \sum_{k=0}^{+\infty}\left(\frac{n}{n+1}\right)^{k}(1+k)^{\beta} .
\end{aligned}
$$

Avec les mêmes arguments que ceux utilisés précédemment, on obtient

$$
\begin{aligned}
\left\|\left(e_{n}-1\right)\left(\alpha^{j}-1\right)\right\|_{\omega} \leq & \frac{1}{n+1} \sum_{k=j}^{-1}\left(\frac{n}{n+1}\right)^{k-j} \omega(k) \\
& +2 \min \left(1,-\frac{j}{n}\right)(n+1)^{\beta} .
\end{aligned}
$$

Grâce aux hypothèses faites sur le poids $\omega$, on a $(1+|j|)^{\beta} \leq \omega(j)$ et $\omega(k) \leq$ $\omega(j)$ si $j \leq k \leq-1$. On en déduit alors que

$$
\left\|\left(e_{n}-1\right)\left(\alpha^{j}-1\right)\right\|_{\omega} \leq \omega(j)+2(1+|j|)^{\beta} \leq 3 \omega(j) .
$$


On vient donc de démontrer que pour tout $j \in \mathbb{Z}$,

$$
\left\|\left(e_{n}-1\right)\left(\alpha^{j}-1\right)\right\|_{\omega} \leq 3 \omega(j),
$$

ce qui entraîne (7). Pour (8), on pose $f_{m}=\sum_{j=-m}^{+m} \widehat{f}(j)\left(\alpha^{j}-1\right)$, de sorte que $\lim _{m \rightarrow+\infty}\left\|f-f_{m}\right\|_{\omega}=0$. On a alors, en utilisant (7),

$$
\begin{aligned}
\left\|\left(e_{n}-1\right) f\right\|_{\omega} & \leq\left\|\left(e_{n}-1\right) f_{m}\right\|_{\omega}+\left\|\left(e_{n}-1\right)\left(f-f_{m}\right)\right\|_{\omega} \\
& \leq\left\|\left(e_{n}-1\right) f_{m}\right\|_{\omega}+3\left\|f-f_{m}\right\|_{\omega} .
\end{aligned}
$$

De (10) et (11), on déduit que pour $j \in \mathbb{Z}, \lim _{n \rightarrow+\infty}\left\|\left(e_{n}-1\right)\left(\alpha^{j}-1\right)\right\|_{\omega}=0$, ce qui entraîne que $\lim _{n \rightarrow+\infty}\left\|\left(e_{n}-1\right) f_{m}\right\|_{\omega}=0$. Il est maintenant facile de voir que $\lim _{n \rightarrow+\infty}\left\|\left(e_{n}-1\right) f\right\|_{\omega}=0$.

Proposition 2.4. Soit $\omega$ un poids satisfaisant $\left(\mathrm{W}_{s}\right)$. On pose

$$
u_{n}=e_{n}^{[s]+1} \quad(n \geq 1),
$$

où $e_{n}$ est la fonction définie en (6). Alors pour toute fonction $f$ dans $A_{\omega}(\mathbb{T})$ telle que $f^{(k)}(1)=0$ pour $0 \leq k \leq[s]$, on a

$$
\lim _{n \rightarrow+\infty}\left\|\left(u_{n}-1\right) f\right\|_{\omega}=0 .
$$

Démonstration. Dans cette démonstration on posera $p=[s]$ et on notera, pour $0 \leq k \leq p, \omega_{k}=\left(\omega_{k}(n)\right)_{n \in \mathbb{Z}}$ la suite définie par

$$
\omega_{k}(n)=\frac{\omega(n)}{(1+|n|)^{k}} \text {. }
$$

En utilisant les relations $e_{n}=n(\alpha-1)\left(e_{n}-1\right)$ et $(\alpha-1) e_{n}^{\prime}=-\left(e_{n}-1\right) e_{n}$ $(n \geq 1)$, on montre par récurrence que pour tout $k$ dans $\{0, \ldots, p\}$, on a

$$
\left(u_{n}-1\right)^{(k)}=\frac{e_{n}-1}{(\alpha-1)^{k}} P_{k}\left(e_{n}\right) \quad(n \geq 1),
$$

où $P_{k}$ est un polynôme de degré inférieur à $k+p$ ne dépendant pas de $n$.

Soit maintenant $f$ dans $A_{\omega}(\mathbb{T})$ telle que $f(1)=0$. D'après le lemme 2.2, il s'agit de montrer que

$$
\begin{aligned}
& \lim _{n \rightarrow+\infty}\left\|\left[\left(u_{n}-1\right) f\right]^{(p)}\right\|_{\omega_{p}}=0, \\
& \lim _{n \rightarrow+\infty}\left[\left(\left(u_{n}-1\right) f\right)^{\wedge}\right](k)=0 \quad(0 \leq k \leq p-1) .
\end{aligned}
$$

Les conditions (16) se montrent facilement à l'aide du théorème de convergence dominée, il reste donc à montrer (15). En utilisant la formule de Leibniz et l'identité (14), on obtient

$$
\begin{aligned}
{\left[\left(u_{n}-1\right) f\right]^{(p)} } & =\sum_{k=0}^{p}\left(\begin{array}{l}
p \\
k
\end{array}\right)\left(u_{n}-1\right)^{(k)} f^{(p-k)} \\
& =\sum_{k=0}^{p}\left(\begin{array}{l}
p \\
k
\end{array}\right)\left(e_{n}-1\right) \frac{f^{(p-k)}}{(\alpha-1)^{k}} P_{k}\left(e_{n}\right) .
\end{aligned}
$$


Or les hypothèses sur le poids $\omega$ nous permettent d'utiliser le lemme 2.2 qui nous assure que

$$
\frac{f^{(p-k)}}{(\alpha-1)^{k}} \in A_{\omega_{p}}(\mathbb{T}) \quad(0 \leq k \leq p) .
$$

De plus, $\omega_{p}$ vérifie la condition $\left(\mathrm{W}_{\beta}\right)$ avec $\beta=s-p$. On déduit alors du lemme 2.3 que si $g \in A_{\omega_{p}}(\mathbb{T})$ et $g(1)=0$, alors $\left\|e_{n} g\right\|_{\omega_{p}} \leq 4\|g\|_{\omega_{p}}(n \geq 1)$. Et comme pour tout $k \in\{0, \ldots, p\}$, la fonction $f^{(p-k)} /(\alpha-1)^{k}$ s'annule en 1 , on peut alors trouver une constante $C>0$ indépendante de $n$ et de $k$ telle que

$$
\left\|\left(e_{n}-1\right) \frac{f^{(p-k)}}{(\alpha-1)^{k}} P_{k}\left(e_{n}\right)\right\|_{\omega_{p}} \leq C\left\|\left(e_{n}-1\right) \frac{f^{(p-k)}}{(\alpha-1)^{k}}\right\|_{\omega_{p}} .
$$

De plus, toujours d'après le lemme 2.3,

$$
\lim _{n \rightarrow+\infty}\left\|\left(e_{n}-1\right) \frac{f^{(p-k)}}{(\alpha-1)^{k}}\right\|_{\omega_{p}}=0 \quad(0 \leq k \leq p) .
$$

Par conséquent, on déduit alors de (17) et (18) que

$$
\lim _{n \rightarrow+\infty}\left\|\left[\left(u_{n}-1\right) f\right]^{(p)}\right\|_{\omega_{p}}=0 .
$$

3. Idéaux fermés de $A_{\omega}(\mathbb{T})$ et de $A_{s}^{+}(\mathbb{T})$. Il est montré dans [2] que si $\omega$ est un poids vérifiant $\left(\mathrm{W}_{s}\right)$ et $(\mathrm{A})$, et $I$ un idéal fermé de $A_{\omega}(\mathbb{T})$ tel que $h^{0}(I)=\left\{z_{0}\right\}$, alors il existe $j$ dans $\{1, \ldots,[s]\}$ tel que $I=\left\{f \in A_{\omega}(\mathbb{T})\right.$ : $\left.f\left(z_{0}\right)=\cdots=f^{(j)}\left(z_{0}\right)=0\right\}$. Nous allons montrer que ce résultat s'étend aux fermés dénombrables. Introduisons d'abord la notation suivante : si $f$ est dans $A_{\omega}(\mathbb{T})$, et $I$ un idéal fermé de $A_{\omega}(\mathbb{T})$, on pose $I(f)=\left\{g \in A_{\omega}(\mathbb{T})\right.$ : $f g \in I\}$. On a le résultat suivant :

Lemme 3.1. Soit $\omega$ un poids vérifiant $\left(\mathrm{W}_{s}\right)$ et $(\mathrm{A})$, et I un idéal fermé de $A_{\omega}(\mathbb{T})$ tel que $h^{0}(I)$ ait un point isolé $z_{0}$. Soit $k=\max \{j \in\{0, \ldots,[s]\}$ : $\left.z_{0} \in h^{j}(I)\right\}$. Alors il existe $g$ dans $I$ de la forme $g=\left(\alpha-z_{0}\right)^{k+1} \psi$, avec $\psi \in A_{\omega}(\mathbb{T})$ et $\psi\left(z_{0}\right) \neq 0$.

Démonstration. Comme $z_{0}$ est un point isolé dans $h^{0}(I)$, il existe $\psi \in$ $A_{\omega}(\mathbb{T})$ telle que

$$
\psi= \begin{cases}1 & \text { sur un voisinage de } z_{0}, \\ 0 & \text { sur un voisinage de } h^{0}(I) \backslash\left\{z_{0}\right\} .\end{cases}
$$

L'algèbre $A_{\omega}(\mathbb{T})$ étant régulière, $1-\psi \in I(\psi)$, ce qui prouve que $h^{0}(I(\psi)) \subset$ $\left\{z_{0}\right\}$. Puisque $\psi \notin I$, on a $I(\psi) \neq A_{\omega}(\mathbb{T})$, et donc $h^{0}(I(\psi))=\left\{z_{0}\right\}$. Par conséquent, comme $\omega$ vérifie les conditions $\left(\mathrm{W}_{s}\right)$ et $(\mathrm{A})$, on déduit de la proposition 6 de [2] qu'il existe un entier $\gamma, 0 \leq \gamma \leq[s]$, tel que

$$
I(\psi)=\left\{f \in A_{\omega}(\mathbb{T}): f\left(z_{0}\right)=\cdots=f^{(\gamma)}\left(z_{0}\right)=0\right\} .
$$


Comme $I \subset I(\psi)$, on a $\gamma \leq k$. D'autre part $\left(\alpha-z_{0}\right)^{\gamma+1}$ appartient à $I(\psi)$, et donc la fonction $g=\left(\alpha-z_{0}\right)^{\gamma+1} \psi$ appartient à $I$. Comme $g^{(k)}\left(z_{0}\right)=0$ et $\psi\left(z_{0}\right) \neq 0$, on a $\gamma \geq k$. Donc $\gamma=k$, et la fonction $g$ ainsi définie convient.

ThÉORÈme 3.2. Soit $\omega$ un poids vérifiant $\left(\mathrm{W}_{s}\right)$ et $(\mathrm{A})$, et I un idéal fermé de $A_{\omega}(\mathbb{T})$ tel que $h^{0}(I)$ est dénombrable. Alors

$$
I=\left\{f \in A_{\omega}(\mathbb{T}): f^{(j)}=0 \operatorname{sur~}^{j}(I)(0 \leq j \leq[s])\right\} .
$$

Démonstration. Soit $I$ un idéal fermé de $A_{\omega}(\mathbb{T})$ tel que $h^{0}(I)$ est dénombrable, et notons $J=\left\{f \in A_{\omega}(\mathbb{T}): f^{(j)}=0 \operatorname{sur}^{j}(I)(0 \leq j \leq[s])\right\}$. L'inclusion $I \subset J$ étant évidente, il reste à montrer l'autre. Soit $f \in J$. Nous allons montrer que $I(f)=A_{\omega}(\mathbb{T})$. Soient $z_{0} \in h^{0}(I) \backslash h^{[s]}(I)$ et $k \in\{0, \ldots,[s]-1\}$ tels que $z_{0} \in h^{k}(I) \backslash h^{k+1}(I)$. Il est facile de voir que $J \subset \overline{\left(\alpha-z_{0}\right)^{k+1} A_{\omega}(\mathbb{T})} \|^{\| \omega}$. Par conséquent, il existe une suite $\left(f_{m}\right)_{m \geq 0}$ de fonctions de la forme $f_{m}=\left(\alpha-z_{0}\right)^{k+1} \phi_{m}$, avec $\phi_{m} \in A_{\omega}(\mathbb{T})$, telle que $\lim _{m \rightarrow+\infty}\left\|f-f_{m}\right\|_{\omega}=0$. De plus, comme $z_{0} \notin h^{[s]}(I)$, le point $z_{0}$ est nécessairement isolé dans $h^{0}(I)$. Donc d'après le lemme précédent, il existe $g$ dans $I$ qui s'écrit $g=\left(\alpha-z_{0}\right)^{k+1} \psi$ avec $\psi \in A_{\omega}(\mathbb{T})$ et $\psi\left(z_{0}\right) \neq 0$. Posons alors

$$
\Psi_{m}=\phi_{m} g=f_{m} \psi \quad(m \geq 0) .
$$

Pour tout entier $m \geq 0$ on a $\Psi_{m} \in I$ et $\lim _{m \rightarrow+\infty}\left\|\Psi_{m}-f \psi\right\|_{\omega}=0$. Comme $I$ est fermé, on a donc $f \psi \in I$, c'est-à-dire $\psi \in I(f)$. Par conséquent, $z_{0} \notin h^{0}(I(f))\left(\operatorname{car} \psi\left(z_{0}\right) \neq 0\right)$. Finalement, on en déduit dans un premier temps que

$$
h^{0}(I(f)) \subset h^{[s]}(I) .
$$

Supposons que $h^{0}(I(f)) \neq \emptyset$; alors $h^{0}(I(f))$ admet un point isolé $\xi_{0}$. Sans perte de généralité, on va supposer que $\xi_{0}=1$. L'algèbre $A_{\omega}(\mathbb{T})$ étant régulière, il existe une fonction $\Phi$ telle que

$$
\Phi= \begin{cases}1 & \text { sur un voisinage de } 1, \\ 0 & \text { sur un voisinage de } h^{0}(I(f)) \backslash\{1\} .\end{cases}
$$

On pose $L_{\Phi}=\left\{h \in A_{\omega}(\mathbb{T}): h \Phi \in I(f)\right\}$, l'idéal de division de $I(f)$ par $\Phi$. On a $1-\Phi \in L_{\Phi}$, et donc $h^{0}\left(L_{\Phi}\right) \subset\{1\}$. Comme $\omega$ vérifie les conditions (A) et $\left(\mathrm{W}_{s}\right)$, on déduit de la proposition 6 de $[2]$ que $\left\{f \in A_{\omega}(\mathbb{T}): f(1)=\right.$ $\left.\cdots=f^{(\gamma)}(1)=0\right\} \subset L_{\Phi}$. Par conséquent, la suite $\left(u_{n}\right)_{n \geq 1}$ définie en (13) appartient à $L_{\Phi}$. D'après l'inclusion $h^{0}(I(f)) \subset h^{[s]}(I)$ établie en (19), on a $f^{(k)}(1)=0$ pour $0 \leq k \leq[s]$. Et comme le poids $\omega$ vérifie la condition $\left(\mathrm{W}_{s}\right)$, on déduit du lemme 2.4 que $\lim _{n \rightarrow+\infty}\left\|\left(u_{n}-1\right) f\right\|_{\omega}=0$. Puisque $u_{n} \Phi f \in I(n \geq 1)$ et $\lim _{n \rightarrow+\infty}\left\|u_{n} \Phi f-\Phi f\right\|_{\omega}=0$, on a $\Phi f \in I$, ce qui contredit le fait que $1 \in h^{0}(I(f))$. Finalement, on a montré que $h^{0}(I(f))=\emptyset$, et donc $I(f)=A_{\omega}(\mathbb{T})$, ce qui signifie exactement que $f \in I$. 
On va maintenant s'intéresser aux idéaux fermés de $A_{s}^{+}(\mathbb{T})$. Puisque l'algèbre $A_{s}(\mathbb{T})$ vérifie la condition de Ditkin analytique forte, le théorème $\mathrm{B}$ de [3] nous assure que tout idéal fermé $I$ de $A_{s}^{+}(\mathbb{T})$ tel que $h_{+}^{0}(I)$ est fini, est de la forme

$$
I=I^{A_{s}} \cap S_{I} \mathcal{H}^{\infty}(\mathbb{D}),
$$

où $I^{A_{s}}$ est l'idéal fermé de $A_{s}(\mathbb{T})$ engendré par $I$. Par conséquent, compte tenu du théorème $3.2, I$ est de la forme

$$
I=\left\{f \in A_{s}^{+}(\mathbb{T}): S_{I} \mid S(f) \text { et } f^{(j)}=0 \text { sur } h^{j}(I) \cap \mathbb{T}(0 \leq j \leq[s])\right\} .
$$

Soit $I$ un idéal fermé non nul de $A_{s}^{+}(\mathbb{T})$. On note

$$
\pi_{s}^{+}: A_{s}^{+}(\mathbb{T}) \rightarrow A_{s}^{+}(\mathbb{T}) / I
$$

la surjection canonique. On a alors le résultat suivant :

Lemme 3.3. Soient $s$ un réel positif et $I$ un idéal fermé non réduit à $\{0\}$ de $A_{s}^{+}(\mathbb{T})$ tel que $S_{I}=1$. Alors

$$
\left\|\pi_{s}^{+}(\alpha)^{-n}\right\|=O\left(e^{\varepsilon \sqrt{n}}\right) \quad(n \rightarrow+\infty) \text { pour tout } \varepsilon>0 .
$$

Démonstration. C'est un résultat établi par A. Atzmon dans la preuve de la proposition 8 de [2] dans le cas où $I=\left\{f \in A_{s}^{+}(\mathbb{T}): f_{\mid E}=0\right\}$, et qui est une conséquence du lemme 5.c de [2] (voir aussi [19, proposition 2.1]). Le résultat ci-dessus se démontre de façon analogue.

Nous avons alors le théorème suivant :

THÉORÈme 3.4. Soient $s$ un réel positif et I un idéal fermé de $A_{s}^{+}(\mathbb{T})$ tel que $S_{I}=1$ et $h_{+}^{0}(I)$ est au plus dénombrable. Alors

$$
I=I\left(1 ; h_{+}^{0}(I), \ldots, h_{+}^{[s]}(I)\right) .
$$

Démonstration. Soit $I$ un idéal fermé sans facteur intérieur de $A_{s}^{+}(\mathbb{T})$ tel que $h_{+}^{0}(I)$ est au plus dénombrable. On déduit du lemme 3.3 que

$$
\left\|\pi_{s}^{+}(\alpha)^{-n}\right\|=O\left(e^{\varepsilon \sqrt{n}}\right) \quad(n \rightarrow+\infty) \text { pour tout } \varepsilon>0 .
$$

On considère alors le poids $\omega$ défini par

$$
\begin{cases}\omega(n)=(1+n)^{s} & (n \geq 0), \\ \omega(-n)=(1+n)^{s} \sup _{0<k \leq n}\left\|\pi_{s}^{+}(\alpha)^{-k}\right\| & (n>0),\end{cases}
$$

et on définit l'application continue $\theta: A_{\omega}(\mathbb{T}) \rightarrow A_{s}^{+}(\mathbb{T}) / I$ par

$$
\theta(f)=\sum_{n=-\infty}^{+\infty} \widehat{f}(n) \pi_{s}^{+}(\alpha)^{n} .
$$

On a $\theta_{\mid A_{s}^{+}(\mathbb{T})}=\pi_{s}^{+}$, et donc

$$
\operatorname{Ker} \theta \cap A_{s}^{+}(\mathbb{T})=I .
$$


Si $I^{A_{\omega}}$ désigne l'idéal fermé de $A_{\omega}(\mathbb{T})$ engendré par $I$, on a

$$
I^{A_{\omega}}=\bar{\bigcup}_{n \geq 0} \alpha^{-n} I{ }^{A_{\omega}(\mathbb{T})} .
$$

Or il est facile de voir que $\alpha^{-n} I \subset \operatorname{Ker} \theta$ pour tout $n \geq 0$, et donc

$$
I^{A_{\omega}} \cap A_{s}^{+}(\mathbb{T}) \subset \operatorname{Ker} \theta \cap A_{s}^{+}(\mathbb{T})=I .
$$

L'autre inclusion étant évidente, on a donc

$$
I=I^{A_{\omega}} \cap A_{s}^{+}(\mathbb{T}) .
$$

Puisque $S_{I}=1$, on a $h_{+}^{k}(I) \subset \mathbb{T}$ pour tout $0 \leq k \leq[s]$. Il est alors facile de voir que $h^{k}\left(I^{A_{\omega}}\right)=h_{+}^{k}(I)$ pour tout $0 \leq k \leq[s]$; on déduit finalement du théorème 3.2 que

$$
I=I\left(1 ; h_{+}^{0}(I), \ldots, h_{+}^{[s]}(I)\right) .
$$

4. Interpolation. On note $\mathcal{C}^{\infty}(\mathbb{T})$ l'ensemble des fonctions de classe $\mathcal{C}^{\infty}$ sur le cercle, que l'on équipe de sa topologie d'espace de Fréchet usuelle définie par les normes $\left(\varrho_{\nu}\right)_{\nu \in \mathbb{R}^{+}}$définies par

$$
\varrho_{\nu}(f)=\sum_{n=-\infty}^{+\infty}|\widehat{f}(n)|(1+|n|)^{\nu} .
$$

On note également

$$
a^{\infty}=a^{\infty}(\mathbb{D})=\left\{f \text { holomorphe dans } \mathbb{D}: f \in \mathcal{C}(\overline{\mathbb{D}}) \text { et } f_{\mid \mathbb{T}} \in \mathcal{C}^{\infty}(\mathbb{T})\right\},
$$

que l'on regardera comme l'ensemble des fonctions de $\mathcal{C}^{\infty}(\mathbb{T})$ à coefficients de Fourier strictement négatifs nuls. Soit $E$ un fermé du cercle unité; on définit

$$
I_{\infty}(E)=\left\{f \in \mathcal{C}^{\infty}(\mathbb{T}): f_{\mid E}^{(i)}=0(i \geq 0)\right\},
$$

et on pose $I_{\infty}^{+}(E)=I_{\infty}(E) \cap a^{\infty}$.

Le dual de $\mathcal{C}^{\infty}(\mathbb{T})$ est $\mathcal{D}^{\prime}(\mathbb{T})$, l'ensemble des distributions sur $\mathbb{T}$. On associe à chaque distribution $T \in \mathcal{D}^{\prime}(\mathbb{T})$ une suite de coefficients de Fourier $(\widehat{T}(n))_{n \in \mathbb{Z}}$, où pour tout entier $n, \widehat{T}(n)=\left\langle\alpha^{-n}, T\right\rangle$ (avec $\left.\alpha: z \mapsto z\right)$, qui vérifient $|\widehat{T}(n)|=O\left(|n|^{m}\right)$ pour un certain entier $m \geq 0$. La dualité entre $\mathcal{C}^{\infty}(\mathbb{T})$ et $\mathcal{D}^{\prime}(\mathbb{T})$ est donnée par la formule

$$
\langle f, T\rangle=\sum_{n=-\infty}^{+\infty} \widehat{f}(n) \widehat{T}(-n) \quad\left(f \in \mathcal{C}^{\infty}(\mathbb{T}), T \in \mathcal{D}^{\prime}(\mathbb{T})\right) .
$$

$I_{\infty}(E)^{\perp}$ (resp. $I_{\infty}^{+}(E)^{\perp}$ ) désigne l'ensemble des distributions s'annulant sur $I_{\infty}(E)$ (resp. sur $\left.I_{\infty}^{+}(E)\right)$. De même $\left(a^{\infty}\right)^{\perp}$ est l'ensemble des distributions s'annulant sur $a^{\infty}$, c'est-à-dire les distributions à coefficients négatifs nuls. 
Dans le cas particulier où le poids $\omega$ est défini par $\omega(n)=(1+n)^{s}$ et $\omega(-n)=(1+n)^{t}$ pour tout $n \geq 0$, avec $t$ et $s$ deux réels, on notera $\left(A_{s, t}(\mathbb{T}),\|\|_{s, t}\right)$ l'algèbre $\left(A_{\omega}(\mathbb{T}),\|\|_{\omega}\right)$. Nous supposerons dorénavant que $t \geq s$, de sorte que $A_{s, t}(\mathbb{T})$ satisfait les conditions $\left(\mathrm{W}_{s}\right)$ et $(\mathrm{A})$. Si $E$ est un fermé de $\mathbb{T}$, on notera

$$
I_{s, t}(E)=\left\{f \in A_{s, t}(\mathbb{T}): f_{\mid E}=\cdots=f_{\mid E}^{([s])}=0\right\},
$$

et $I_{s}^{+}(E)=I_{s, t}(E) \cap A_{s}^{+}(\mathbb{T})$. On identifiera le dual de $A_{s, t}(\mathbb{T})$ (que l'on note $\left.\left(A_{s, t}(\mathbb{T})\right)^{\prime}\right)$ au sous-espace de $\mathcal{D}^{\prime}(\mathbb{T})$ formé des distributions $T$ telles que

$$
\sup _{n \leq 0} \frac{|\widehat{T}(n)|}{(1+|n|)^{s}}+\sup _{n>0} \frac{|\widehat{T}(n)|}{(1+n)^{t}}<+\infty .
$$

$I_{s, t}(E)^{\perp}\left(\right.$ resp. $\left.I_{s}^{+}(E)^{\perp}\right)$ désigne l'ensemble des éléments de $\left(A_{s, t}(\mathbb{T})\right)^{\prime}$ s'annulant sur $I_{s, t}(E)$ (resp. sur $I_{s}^{+}(E)$ ). De même $A_{s}^{+}(\mathbb{T})^{\perp}$ est l'ensemble des éléments de $\left(A_{s, t}(\mathbb{T})\right)^{\prime}$ s'annulant sur $A_{s}^{+}(\mathbb{T})$, c'est-à-dire les éléments de $\left(A_{s, t}(\mathbb{T})\right)^{\prime}$ à coefficients négatifs nuls.

DÉfinition 4.1. Soient $s$ et $t$ deux réels positifs tels que $t \geq s$. On dira qu'un fermé $E$ du cercle unité est d'interpolation pour $A_{s, t}(\mathbb{T})$ si

$$
\forall f \in A_{s, t}(\mathbb{T}), \exists g \in A_{s}^{+}(\mathbb{T}): \quad f_{\mid E}^{(i)}=g_{\mid E}^{(i)} \quad(0 \leq i \leq[s]),
$$

c'est-à-dire si $A_{s}^{+}(\mathbb{T})+I_{s, t}(E)=A_{s, t}(\mathbb{T})$. On dira qu'un fermé $E$ du cercle unité est d'interpolation pour $a^{\infty}$ si

$$
\forall f \in \mathcal{C}^{\infty}(\mathbb{T}), \exists g \in a^{\infty}: \quad f_{\mid E}^{(i)}=g_{\mid E}^{(i)} \quad(i \geq 0),
$$

c'est-à-dire si $a^{\infty}+I_{\infty}(E)=\mathcal{C}^{\infty}(\mathbb{T})$.

H. Alexander, B. A. Taylor et D. L. Williams [1] ont donné une caractérisation géométrique des ensembles d'interpolation pour $a^{\infty}$. Ils ont montré qu'un fermé du cercle unité est d'interpolation pour $a^{\infty}$ si et seulement si $E$ vérifie la condition (ATW). On a également la caractérisation suivante de ces ensembles:

Proposition 4.1. Soit E un fermé du cercle unité. Alors les propriétés suivantes sont équivalentes:

(i) $E$ est un ensemble d'interpolation pour $a^{\infty}$.

(ii) $I_{\infty}^{+}(E)^{\perp}=I_{\infty}(E)^{\perp}+\left(a^{\infty}\right)^{\perp}$.

(iii) Pour tout $s \geq 0$, il existe une constante $C>0$ et $t \geq 0$ tels que

$$
\sup _{n \in \mathbb{Z}} \frac{|\widehat{T}(n)|}{(1+|n|)^{t}} \leq C \sup _{n \leq 0} \frac{|\widehat{T}(n)|}{(1+|n|)^{s}} \quad\left(T \in I_{\infty}(E)^{\perp}\right) .
$$

Démonstration. L'équivalence (i) $\Leftrightarrow$ (ii) a été établie dans $[1$, proposition 2.1]. On va achever la preuve en prouvant que (i) $\Leftrightarrow$ (iii). On sait que $E$ est 
d'interpolation pour $a^{\infty}$ si et seulement si l'injection canonique

$$
i: a^{\infty} / I_{\infty}^{+}(E) \rightarrow \mathcal{C}^{\infty}(\mathbb{T}) / I_{\infty}(E)
$$

est surjective. Puis en utilisant la proposition 4 du paragraphe IV.30 de [4], qui caractérise les surjections entre espaces de Fréchet, on déduit que $E$ est d'interpolation pour $a^{\infty}$ si et seulement si pour tout $s \geq 0$, il existe une constante $C>0$ et $t \geq 0$ tels que pour tout $T$ dans $I_{\infty}(E)^{\perp}$,

$\left(|\langle f, T\rangle| \leq \varrho_{s}(f), f \in a^{\infty} / I_{\infty}^{+}(E)\right) \Rightarrow\left(|\langle f, T\rangle| \leq C \varrho_{t}(f), f \in \mathcal{C}^{\infty}(\mathbb{T}) / I_{\infty}(E)\right)$, c'est-à-dire si et seulement si pour tout $s \geq 0$, il existe une constante $C>0$ et $t \geq 0$ tels que

$$
\left(\sup _{n \leq 0} \frac{|\widehat{T}(n)|}{(1+|n|)^{s}} \leq 1, T \in I_{\infty}(E)^{\perp}\right) \Rightarrow \sup _{n \in \mathbb{Z}} \frac{|\widehat{T}(n)|}{(1+|n|)^{t}} \leq C,
$$

ce qui est clairement équivalent à (iii).

On a également un résultat analogue concernant les ensembles d'interpolation pour $A_{s, t}(\mathbb{T})(t \geq s)$ :

Proposition 4.2. Soit $E$ un fermé du cercle unité et $s, t$ deux réels positifs tels que $t \geq s$. Alors les propriétés suivantes sont équivalentes :

(i) $E$ est un ensemble d'interpolation pour $A_{s, t}(\mathbb{T})$.

(ii) $I_{s}^{+}(E)^{\perp}=I_{s, t}(E)^{\perp}+A_{s}^{+}(\mathbb{T})^{\perp}$.

(iii) Il existe une constante $C>0$ telle que

$$
\sup _{n>0} \frac{|\widehat{T}(n)|}{(1+|n|)^{t}}+\sup _{n \leq 0} \frac{|\widehat{T}(n)|}{(1+|n|)^{s}} \leq C \sup _{n \leq 0} \frac{|\widehat{T}(n)|}{(1+|n|)^{s}} \quad\left(T \in I_{s, t}(E)^{\perp}\right) .
$$

On est maintenant en mesure d'énoncer un résultat qui fait le lien entre les ensembles d'interpolation pour $a^{\infty}$ et les ensembles d'interpolation pour $A_{s, t}(\mathbb{T})(t \geq s)$.

ThÉORÈme 4.3. Soit $E$ un fermé du cercle unité $\mathbb{T}$.

(i) Si $E$ est d'interpolation pour $a^{\infty}$, alors pour tout $s \geq 0$, il existe $t \geq s$ tel que $E$ soit d'interpolation pour $A_{s, t}(\mathbb{T})$.

(ii) Réciproquement, si $E$ est dénombrable et si pour tout $s \geq 0$, il existe $t \geq s$ tel que $E$ soit d'interpolation pour $A_{s, t}(\mathbb{T})$, alors $E$ est d'interpolation pour $a^{\infty}$.

Démonstration. (i) Cette assertion découle directement des caractérisations (iii) des propositions 4.1 et 4.2 puisque $I_{s, t}(E)^{\perp}$ s'injecte dans $I_{\infty}(E)^{\perp}$.

(ii) Supposons que $E$ vérifie les hypothèses de l'assertion (ii); on va montrer que $E$ satisfait la propriété (iii) de la proposition 4.1. Soit $s$ un réel positif; il existe $t \geq s$ tel que $E$ soit d'interpolation pour $A_{s, t}(\mathbb{T})$. Donc 
d'après la proposition 4.2 , il existe une constante $C>0$ telle que pour tout élément de $I_{s, t}(E)^{\perp}$,

$$
\sup _{n>0} \frac{|\widehat{T}(n)|}{(1+|n|)^{t}} \leq C \sup _{n \leq 0} \frac{|\widehat{T}(n)|}{(1+|n|)^{s}} .
$$

Soit alors $T$ un élément de $I_{\infty}(E)^{\perp}$. Si $\sup _{n \leq 0}|\widehat{T}(n)| /(1+|n|)^{s}=+\infty$, alors $T$ vérifie trivialement (22).

Supposons donc que $\sup _{n \leq 0}|\widehat{T}(n)| /(1+|n|)^{s}<+\infty$. Il existe alors $t^{\prime} \geq t$ tel que $T$ soit dans $\left(A_{s, t^{\prime}}(\mathbb{T})\right)^{\prime}$. Notons $K$ la fermeture de $I_{\infty}(E)$ dans $A_{s, t^{\prime}}(\mathbb{T})$. On vérifie facilement que $K$ est un idéal fermé de $A_{s, t^{\prime}}(\mathbb{T})$ tel que $h(K) \subset E$, et ainsi d'après le théorème $3.2, I_{s, t^{\prime}}(E) \subset K$. L'inclusion inverse étant acquise, on a finalement $K=I_{s, t^{\prime}}(E)$. Et comme $T$ est dans $I_{\infty}(E)^{\perp}$, $T$ appartient à $I_{s, t^{\prime}}(E)^{\perp}$ par continuité de $T$ sur $A_{s, t^{\prime}}(\mathbb{T})$. Mais puisque $E$ est d'interpolation pour $A_{s, t}(\mathbb{T})$, les inclusions naturelles

$$
A_{s}^{+}(\mathbb{T}) / I_{s}^{+}(E) \subset A_{s, t^{\prime}}(\mathbb{T}) / I_{s, t^{\prime}}(E) \subset A_{s, t}(\mathbb{T}) / I_{s, t}(E)
$$

sont en réalité des égalités. Par conséquent, leurs duaux sont égaux et donc $T \in I_{s, t}(E)^{\perp}$. Ainsi $T$ vérifie la propriété $(22)$, ce qui achève la démonstration.

\section{Interpolation dans $A_{s, t}(\mathbb{T})$ et opérateurs}

Proposition 5.1. Soit $E$ un fermé du cercle unité, $s$ et $t$ deux réels positifs tels que $t \geq s$.

(i) Si on suppose que $E$ est dénombrable et d'interpolation pour $A_{s, t}(\mathbb{T})$, alors $E$ vérifie la propriété $P(s, t, E)$.

(ii) Réciproquement, si $E$ vérifie la condition de Carleson (C) et la propriété $P(s, t, E)$, alors $E$ est d'interpolation pour $A_{s, t}(\mathbb{T})$.

Démonstration. (i) Soit $T$ un opérateur inversible sur un espace de Banach $X$ vérifiant les conditions (1) et (2). On définit alors le poids $\omega$ par

$$
\begin{cases}\omega(n)=(1+n)^{s} & (n \geq 0) \\ \omega(-n)=(1+n)^{s} \sup _{0<k \leq n}\left\|T^{-k}\right\| & (n>0) .\end{cases}
$$

Puisque $T$ vérifie la condition (1), on peut définir un opérateur borné $\Phi$ de $A_{\omega}(\mathbb{T})$ dans $\mathcal{L}(X)$ par

$$
\Phi(f)=f(T)=\sum_{n=-\infty}^{+\infty} \widehat{f}(n) T^{n} \quad\left(f \in A_{\omega}(\mathbb{T})\right) .
$$

Puisque $A_{\omega}(\mathbb{T})$ est régulière, on a $h(\operatorname{Ker} \Phi)=\operatorname{Sp} T \subset E$ (voir [7, théorème $2.5])$, et donc $\left\{f \in A_{\omega}(\mathbb{T}): f_{\mid E}=\cdots=f_{\mid E}^{([s])}=0\right\} \subset \operatorname{Ker} \Phi$ d'après le théorème 3.2. En utilisant le fait que $E$ est d'interpolation pour $A_{s, t}(\mathbb{T})$ 
et des méthodes similaires à celles utilisées dans [20] pour la preuve du théorème 2.6, on montre que, pour $n \geq 0$,

$$
\left\|T^{-n}\right\| \leq C\left\|\alpha^{-n}\right\|_{s, t}=C(1+n)^{t},
$$

ce qui prouve que $T$ vérifie la propriété (3).

(ii) Pour la réciproque, on considère l'opérateur $T$ défini sur $A_{s}^{+}(\mathbb{T}) / I_{s}^{+}(E)$ par

$$
T: \pi_{s}^{+}(f) \mapsto \pi_{s}^{+}(\alpha f) \quad\left(f \in A_{s}^{+}(\mathbb{T})\right),
$$

où $\pi_{s}^{+}$est la surjection canonique de $A_{s}^{+}(\mathbb{T}) \operatorname{sur} A_{s}^{+}(\mathbb{T}) / I_{s}^{+}(E)$. On a $\left\|T^{n}\right\|=$ $O\left(n^{s}\right)(n \rightarrow+\infty)$. Or, puisque $E$ est de Carleson, $I_{s}^{+}(E)$ est non réduit à $\{0\}$ (voir [5]). De plus, $I_{s}^{+}(E)$ est sans facteur intérieur, donc en utilisant le lemme 3.3, on obtient l'évaluation

$$
\left\|T^{-n}\right\|=O\left(e^{\varepsilon \sqrt{n}}\right) \quad(n \rightarrow+\infty) \text { pour tout } \varepsilon>0 .
$$

Et puisque $\operatorname{Sp} T=\operatorname{Sp} \pi_{s}^{+}(\alpha)=E$, on a

$$
\left\|T^{-n}\right\|=\left\|\pi_{s}^{+}(\alpha)^{-n}\right\|=O\left(n^{t}\right) \quad(n \rightarrow+\infty),
$$

ce qui montre que $E$ est un ensemble d'interpolation pour $A_{s, t}(\mathbb{T})$.

Le résultat suivant, annoncé dans l'introduction, est alors une conséquence directe du théorème 4.3 et de la proposition 5.1.

ThÉORÈme 5.2. Soit E un fermé dénombrable du cercle unité $\mathbb{T}$. Alors les conditions suivantes sont équivalentes:

(i) E vérifie la condition (ATW).

(ii) $E$ vérifie la condition $(\mathrm{C})$, et pour tout $s \geq 0$, il existe $t \geq s$ tel que la propriété $P(s, t, E)$ soit vérifiée.

Nous allons conclure en montrant que les hypothèses du théorème sont optimales. Soit $E$ un ensemble fermé du cercle unité et $\mu$ une mesure à support dans $E$. Soit $J_{\mu}$ la fonction singulière associée à $\mu$, à savoir

$$
J_{\mu}(z)=\exp \left\{-\frac{1}{2 \pi} \int_{0}^{2 \pi} \frac{e^{i t}+z}{e^{i t}-z} d \mu(t)\right\} \quad(|z|<1) .
$$

On pose $\mathcal{H}_{0}=\mathcal{H}^{2}(\mathbb{D}) \ominus J_{\mu} \mathcal{H}^{2}(\mathbb{D})$, où $\mathcal{H}^{2}(\mathbb{D})$ désigne l'espace de Hardy usuel. On note $P_{\mathcal{H}_{0}}$ la projection orthogonale sur $\mathcal{H}_{0}$ et $\alpha: z \mapsto z$. On définit alors l'opérateur $T_{\mu}$ sur $\mathcal{H}_{0}$ par

$$
T_{\mu}(f)=P_{\mathcal{H}_{0}}(\alpha f) \quad\left(f \in \mathcal{H}_{0}\right) .
$$

D'après [15, proposition 5.1, p. 117], on a $\operatorname{Sp} T_{\mu}=\operatorname{Supp} \mu \subset E$.

Si on remplace la condition "pour tout $\varepsilon>0$ " dans la propriété $P(s, t, E)$ par une condition à $\varepsilon$ fixé, alors la propriété $P(0, t,\{1\})$ n'est vérifiée pour aucun réel $t \geq 0$. En effet, soient $\varepsilon_{0}>0, \mu=2 \pi \varepsilon_{0}^{2} \delta_{1}$ (où $\delta_{1}$ est la mesure de 
Dirac en 1) et $T_{1}=T_{\mu}$ l'opérateur défini en (24). Alors $T_{1}$ est un opérateur non unitaire tel que $\operatorname{Sp} T_{1}=\{1\}$ et

$$
\left\|T_{1}^{-n}\right\|=O\left(e^{4 \varepsilon_{0} \sqrt{n}}\right) \quad(n \rightarrow+\infty)
$$

(voir [20] pour plus de détails). Le théorème 6.4 de [6] nous montre alors que $T_{1}^{-n}=O\left(n^{t}\right)(n \rightarrow+\infty)$ n'est satisfait pour aucun réel $t \geq 0$.

Nous allons maintenant montrer que l'hypothèse de dénombrabilité de $E$ dans le théorème 5.2 est essentielle. Pour cela, on a besoin du lemme suivant :

Lemme 5.3. Tout fermé non dénombrable et de mesure nulle du cercle unité contient un ensemble parfait qui vérifie la condition de Carleson $(\mathrm{C})$.

Démonstration. Soit $S$ un fermé non dénombrable et de mesure nulle du cercle unité. Sans perte de géneralité, on peut supposer que $1 \notin S$. On écrit alors $S=\left\{e^{i t}: t \in E\right\}$, où $E$ est un fermé de $\mathbb{R}$ inclu dans $] 0,2 \pi[$, et on se ramène ainsi à la droite réelle. Soit $P$ la partie parfaite de $E$. On pose

$$
P_{0}=\{x \in P: \exists \varepsilon>0 \text { tel que }] x-\varepsilon, x[\cap P=\emptyset \text { ou }] x, x+\varepsilon[\cap P=\emptyset\},
$$

c'est-à-dire l'ensemble des points de $P$ qui sont limites d'un seul côté d'une suite de points de $P$. Montrons dans un premier temps que $P_{0}$ est au plus dénombrable. On pose pour cela

$$
Q_{n}=\left\{x \in P_{0}:\right] x-1 / n, x[\cap P=\emptyset \text { ou }] x, x+1 / n[\cap P=\emptyset\} \quad(n \geq 1) .
$$

Il est clair que $P_{0}=\bigcup_{n=1}^{+\infty} Q_{n}$ et que chaque $Q_{n}$ est fini. Par conséquent, $P_{0}$ est au plus dénombrable.

Soient $a_{0}$ et $b_{0}$ deux points de $P \backslash P_{0}$ tels que $a_{0}<b_{0}$ et $\left|a_{0}-b_{0}\right| \leq 1$; on pose $I_{0}=\left[a_{0}, b_{0}\right]$. À la première étape, on retire de $I_{0}$ un intervalle ouvert $J_{1}^{(1)}$ dont les extrémités sont dans $P \backslash P_{0}$, de sorte qu'il reste deux intervalles fermés $I_{1}^{(1)}$ et $I_{2}^{(1)}$ qui soient non vides, non réduits à un singleton et de longueur inférieure à $\frac{1}{3}\left(b_{0}-a_{0}\right.$ ) (un tel choix est possible car $P_{0}$ est au plus dénombrable). On note $F_{1}$ l'ensemble constitué des deux intervalles fermés $I_{1}^{(1)}$ et $I_{2}^{(1)}$. Le fait que les extrémités de ces deux intervalles soient dans $P \backslash P_{0}$ permet de réitérer le procédé sur chacun d'eux.

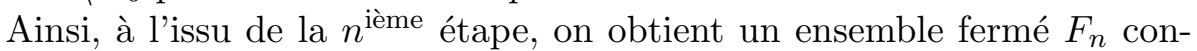
stitué de $2^{n}$ intervalles fermés de longueur inférieure à $3^{-n}\left(b_{0}-a_{0}\right)$, en ayant retiré de nouveau $2^{n-1}$ intervalles ouverts $J_{k}^{(n)}\left(1 \leq k \leq 2^{n-1}\right)$ à extrémités dans $P \backslash P_{0}$. On pose alors

$$
F=\bigcap_{n=1}^{+\infty} F_{n} .
$$

$F$ est clairement un ensemble parfait contenu dans $P$. Il reste à vérifier que $F$ satisfait la condition de Carleson (C). On a $I_{0} \backslash F=\bigcup_{n=1}^{+\infty} \bigcup_{k=1}^{2^{n-1}} J_{k}^{(n)}$. 
Comme $F$ est de mesure nulle, il satisfait la condition de Carleson si et seulement si

$$
\sum_{n=1}^{+\infty} \sum_{k=1}^{2^{n-1}}\left|J_{k}^{(n)}\right| \log \frac{1}{\left|J_{k}^{(n)}\right|}<+\infty .
$$

Or chaque intervalle $J_{k}^{(n)}$ est de longueur inférieure à $3^{-(n-1)}\left(b_{0}-a_{0}\right)$. Comme la fonction $x \mapsto x \log x^{-1}$ est croissante sur $\left[0, e^{-1}\right]$, pour $n$ assez grand, on a

$$
\begin{aligned}
\sum_{k=1}^{2^{n-1}}\left|J_{k}^{(n)}\right| \log \frac{1}{\left|J_{k}^{(n)}\right|} & \leq 2^{n-1} \frac{b_{0}-a_{0}}{3^{n-1}} \log \frac{3^{n-1}}{b_{0}-a_{0}} \\
& \leq\left(b_{0}-a_{0}\right)\left((n-1) \log 3-\log \left(b_{0}-a_{0}\right)\right)\left(\frac{2}{3}\right)^{n-1}
\end{aligned}
$$

et par conséquent (25) a bien lieu.

Proposition 5.4. Soit E un ensemble fermé non dénombrable du cercle unité. Alors la propriété $P(0, t,\{1\})$ n'est vérifiée pour aucun réel $t \geq 0$.

Démonstration. Soit $E$ un ensemble fermé non dénombrable du cercle unité. D'après le lemme précédent, $E$ contient un ensemble parfait $F$ qui vérifie la condition de Carleson. Soit alors $\mu$ une mesure continue à support inclu dans $F$ et $T_{\mu}$ l'opérateur défini en (24). $T_{\mu}$ est une contraction dont le spectre est inclu dans $F$, et on montre en utilisant le lemme 2 de [2] que

$$
\left\|T_{\mu}^{-n}\right\|=O\left(e^{\varepsilon \sqrt{n}}\right) \quad(n \rightarrow+\infty) \text { pour tout } \varepsilon>0 .
$$

Maintenant on conclut par des arguments bien connus (voir [9]) que $\lim _{n \rightarrow+\infty}\left\|T_{\mu}^{-n}\right\|=+\infty$, et donc que $T_{\mu}$ n'est pas unitaire. Puis on déduit du théorème 6.4 de [6] que $T_{\mu}^{-n}=O\left(n^{t}\right)(n \rightarrow+\infty)$ n'est satisfait pour aucun réel $t \geq 0$.

\section{Références}

[1] H. Alexander, B. A. Taylor and D. L. Williams, The interpolating sets for $\mathcal{A}^{\infty}$, J. Math. Anal. Appl. 36 (1971), 556-566.

[2] A. Atzmon, Operators which are annihilated by analytic functions and invariant subspaces, Acta Math. 144 (1980), 27-63.

[3] C. Bennett and J. E. Gilbert, Homogeneous algebras on the circle. I. Ideals of analytic functions, Ann. Inst. Fourier (Grenoble) 22 (1972), no. 3, 1-19.

[4] N. Bourbaki, Éléments de mathématique. Espaces vectoriels topologiques. Chapitres 1 à 5, Masson, Paris, 1981.

[5] L. Carleson, Sets of uniqueness for functions regular in the unit circle, Acta Math. 87 (1952), 325-345. 
[6] J. Esterle, Uniqueness, strong forms of uniqueness and negative powers of contractions, dans : Banach Center Publ. 30, Inst. Math. Polish Acad. Sci., Warszawa, 1994, 127-145.

[7] J. Esterle, E. Strouse and F. Zouakia, Theorems of Katznelson-Tzafriri type for contractions, J. Funct. Anal. 94 (1990), 273-287.

[8] - - - - Closed ideals of $A^{+}$and the Cantor set, J. Reine Angew. Math. 449 (1994), 65-79.

[9] J. Esterle, M. Zarrabi and M. Rajoelina, On contractions with spectrum contained in the Cantor set, Math. Proc. Cambridge Philos. Soc. 117 (1995), 339-343.

[10] K. Hoffman, Banach Spaces of Analytic Functions, Prentice-Hall, Englewood Cliffs, NJ, 1962.

[11] J.-P. Kahane, Idéaux primaires fermés dans certaines algèbres de Banach de fonctions analytiques, dans : L'analyse harmonique dans le domaine complexe (Actes Table Ronde Internat. CNRS, Montpellier, 1972), Lecture Notes in Math. 336, Springer, 1973, 5-14.

[12] Y. Katznelson, An Introduction to Harmonic Analysis, Wiley, New York, 1968.

[13] B. I. Korenblum, Closed ideals of the ring $A^{n}$, Funktsional. Anal. i Prilozhen. 6 (1972), 38-53 (in Russian); English transl.: Functional Anal. Appl. 6 (1972), 203-214.

[14] A. L. Matheson, Closed ideals in rings of analytic functions satisfying a Lipschitz condition, in: Lecture Notes in Math. 604, Springer, Berlin, 1977, 67-72.

[15] B. Sz.-Nagy and C. Foiaş, Analyse harmonique des opérateurs de l'espace de Hilbert, Akadémiai Kiadó, Budapest, 1967.

[16] W. Rudin, The closed ideals in an algebra of analytic functions, Canad. J. Math. 9 (1957), 426-434.

[17] B. A. Taylor and D. L. Williams, Ideals in rings of analytic functions with smooth boundary values, ibid. 22 (1970), 1266-1283.

[18] - - - Zeros of Lipschitz functions analytic in the unit disc, Michigan Math. J. 18 (1971), 129-139.

[19] M. Zarrabi, Synthèse spectrale dans certaines algèbres de Beurling sur le cercle unité, Bull. Soc. Math. France 118 (1990), 241-249.

[20] - Contractions à spectre dénombrable et propriétés d'unicité des fermés dénombrables du cercle, Ann. Inst. Fourier (Grenoble) 43 (1993), 251-263.

Université Bordeaux I

351, cours de la Libération

33405 Talence Cedex, France

E-mail: Cyril.Agrafeuil@math.u-bordeaux1.fr

Received May 14, 2003

Revised version June 1, 2004 